\title{
Actualización y raciocinio del mejor tratamiento antihipertensivo
}

\author{
Update and reasoning out the best antihypertensive treatment
}

\author{
Alfonso Bryce-Moncloa $1,2,3,4,5,6$ \\ ${ }^{1}$ Docente de Posgrado en Medicina y Cardiología, Facultad de Medicina, Universidad Nacional Mayor de San Marcos. \\ ${ }^{2}$ Docente de Posgrado en Medicina y Cardiología, Facultad de Medicina, Universidad Peruana Cayetano Heredia. \\ ${ }^{3}$ Director del Departamento de Cardiología e Investigación Clínica de Cardiogolf/Clínica El Golf. \\ ${ }^{4}$ Past-Presidente de la Sociedad Peruana de Hipertensión Arterial. \\ ${ }^{5}$ Miembro del II Consenso Latinoamericano de Hipertensión. \\ ${ }^{6}$ Vocal del Colegio Panamericano de Endotelio.
}

\section{Resumen}

La evolución en el manejo de la hipertensión arterial ha variado, desde la monoterapia con dosis altas de diuréticos, recomendada por el Joint National Committee I (JNC), en 1977, hasta la terapia combinada a dosis bajas de medicamentos, aconsejada por el JNC VII, en el 2003. Se hace una revisión del progreso de estas terapias antihipertensivas y las recomendaciones actuales.

Palabras clave: Hipertensión, terapéutica; agentes antihipertensivos; agentes vasodilatadores.

\begin{abstract}
Evolution in treatment of arterial hypertension has changed from monotherapy with high doses of diuretics as recommended by the "Joint National Committee I" (JNC) in 1977 to low doses of combined therapy as suggested by the JNC VII in 2003. A review of progress in antihypertensive therapies and current recommendations is done.

Keywords: Hypertension, therapy; antihypertensive, agents; vasodilatador, agents.
\end{abstract}

An Fac med. 2010;71 (4):251-5
La evolución en el manejo de la hipertensión arterial ha sido cambiante ${ }^{(1,2)}$, desde la monoterapia con altas dosis de diuréticos ${ }^{(3)}$, recomendada por el "Joint National Committee I" (JNC) ${ }^{(4)}$, en 1977, hasta la terapia combinada a dosis bajas de medicamentos ${ }^{(5-9)}$, recomendada por el JNC VII ${ }^{(10,11)}$, en el año 2003. Se espera para el próximo año las recomendaciones del JNC VIII ${ }^{(12)}$ (figura 1).
El desarrollo de las terapias antihipertensivas ha progresado ${ }^{(13)}$, siendo los últimos medicamentos registrados los bloqueadores del sistema renina angiotensina aldosterona (SRAA) (figura 2).

\section{¿CUÁL ES EL MEJOR TRATAMIENTO?}

A la pregunta ¿Cuál es el mejor tratamiento?, debemos considerar las po-

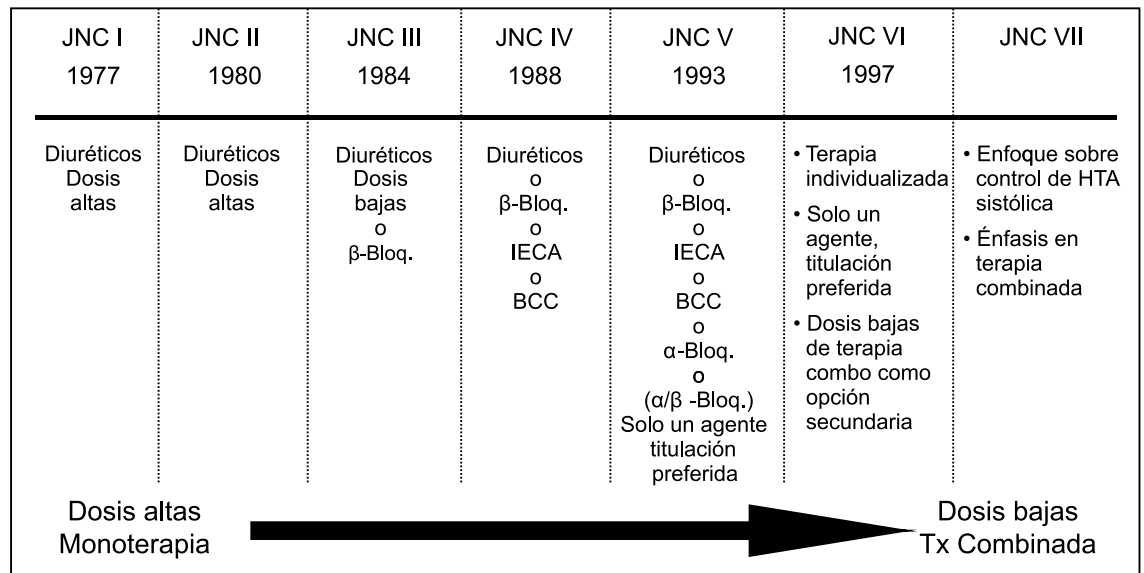

Figura 1. Evolución en el manejo de la hipertensión arterial. blaciones especiales, daño en órganos blanco, cuadros especiales, de manera de poder escoger el mejor tratamiento, como lo observamos en la figura $3^{(14-18)}$.

\section{¿CUÁNDO INICIAR EL TRATAMIENTO}

A la pregunta cuándo iniciar el tratamiento, debemos considerar, de acuerdo a las Guías Latinoamericanas de $\mathrm{Hi}$ pertensión ${ }^{(19)}$, las condiciones sociales con múltiples factores de riesgo, como síndrome metabólico, daño en órgano blanco, diabetes mellitus que, de acuerdo a las cifras de presión arterial y estratificación del riesgo, serían indicaciones para considerar evaluar el tratamiento farmacológico (tabla 1$)^{(19)}$.

A las preguntas ¿cuál tratamiento? Y itodos los fármacos son iguales? (ver tabla 2), estos deben ser considerados de acuerdo a la mejor evidencia clínica, en relación al daño subclínico a órgano blanco, eventos clínicos, condiciones clínicas asociadas, entre otros (tabla 3) ${ }^{(20)}$.

De acuerdo al JNC 7, el algoritmo para el tratamiento de hipertensión arterial, 


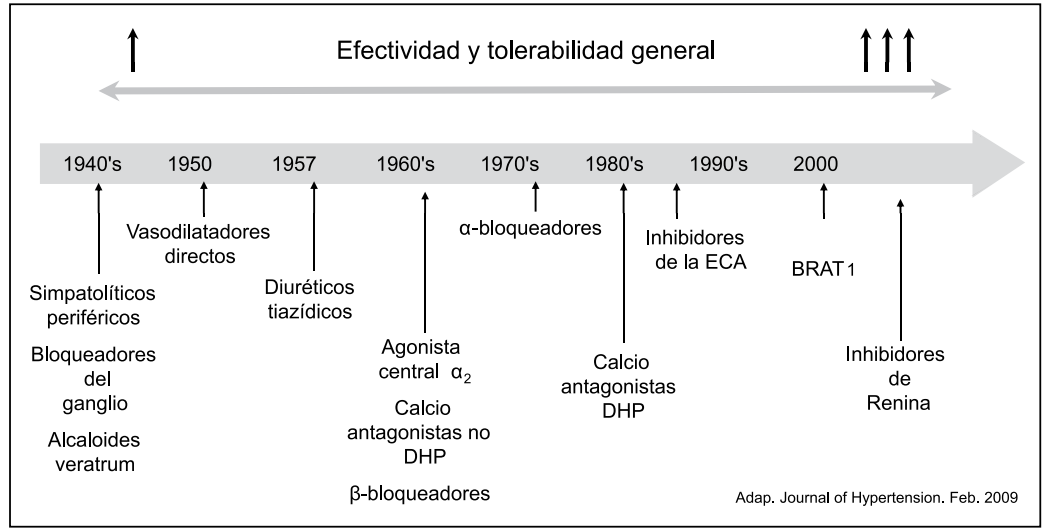

Figura 2. Desarrollo de las terapias antihipertensivas.

\begin{tabular}{|c|c|c|}
\hline \multirow{2}{*}{$\begin{array}{l}\text { Poblaciones } \\
\text { especiales }\end{array}$} & Daño OB & \multirow[b]{2}{*}{ Cuadros especiales } \\
\hline & & \\
\hline Blancos & cerebro & \multirow{3}{*}{$\begin{array}{l}\text { Nuevos FxRx } \\
\text { Microalbuminuria;hiperuricemia; } \\
\text { IMT (grosor-íntima carotídeo) }\end{array}$} \\
\hline Negros & Corazón & \\
\hline \multirow{2}{*}{$\begin{array}{l}\text { Adulto mayor } \\
\text { (Viejos-HSA) }\end{array}$} & Riñon & \\
\hline & EVP & D. Eréctil \\
\hline S. Metabólico & \multirow[t]{3}{*}{ Retinopatía } & Bata blanca \\
\hline Diabetes & & Estrés \\
\hline I. R. (Nefrópatas) & & Antiinflamatorios; [ a-bloq. ] \\
\hline HVI & & Obesidad \\
\hline \multirow[t]{2}{*}{ Embarazo } & & $\mathrm{HVl}$; Angina crónica estable \\
\hline & & Asma-EPOC \\
\hline
\end{tabular}

Figura 3. ¿Cuál es el mejor tratamiento para la hipertensión arterial? primero es la modificación del estilo de vida y, luego, de acuerdo a la estratificación del riesgo y estadio de hipertensión, el tratamiento es farmacológico. El JNC VII tiene preferencia por los diuréticos. Probablemente el JNCVIII planteará las siguientes interrogantes: ¿Por qué tiazida para la mayoría? ¿Tratamiento combinado temprano? ¿Bloqueo del sistema renina angiotensina para la mayoría?, indicando la hipótesis de tratamiento temprano y simplificado, como estrategia (figura 4) ${ }^{(10)}$.

Se muestra las combinaciones terapéuticas eficaces ${ }^{(21)}$ en la figura 5, donde la línea continua muestra la mejor asociación de fármacos ${ }^{(22)}$.

La combinación de betabloqueadores con diuréticos predispone a la aparición de nuevos casos de diabetes y/o dislipidemia $^{(23,24)}$, por lo que no debería utilizarse en pacientes con síndrome metabólico o predisposición para la diabetes (tabla 4). En caso estén indicados, considerar los que tienen efecto vasodilatador. Se debería asociar a fármacos considerados 'protectores', como los bloqueadores del sistema renina angiotensina (tabla 5$)^{(25)}$.

Recientemente, la Sociedad Americana de Hipertensión (ASH) recomienda algunas asociaciones de medicamentos (tabla 6), con algunos comentarios al respecto (tabla 7$)^{(26)}$.

Tabla 1. Consenso Latinoamericano de HTA - Estratificación del riesgo.

\begin{tabular}{|c|c|c|c|c|c|c|}
\hline $\begin{array}{l}\text { Otros FRC } \\
\text { o enfermedades }\end{array}$ & $\begin{array}{l}\text { PA normal óptima } \\
(\leq 120 / 80)\end{array}$ & $\begin{array}{l}\text { PA normal } \\
(\leq 130 / 85)\end{array}$ & $\begin{array}{l}\text { PA normal alta } \\
(130-139 / 85-89)\end{array}$ & $\begin{array}{l}\text { HTA grado } 1 \\
(140-149 / 90-99)\end{array}$ & $\begin{array}{l}\text { HTA grado } 2 \\
(160-179 / 100-109)\end{array}$ & $\begin{array}{l}\text { HTA grado } 3 \\
(\geq 180 \geq 110)\end{array}$ \\
\hline No otros FR & Riesgo promedio & Riesgo bajo & $\begin{array}{l}\text { Incremento de } \\
\text { riesgo }\end{array}$ & Riesgo agregado & $\begin{array}{l}\text { Riesgo agregado } \\
\text { moderado }\end{array}$ & $\begin{array}{l}\text { Riesgo agregado } \\
\text { alto }\end{array}$ \\
\hline $\begin{array}{l}\text { 1-2 FR y } \\
\text { Condiciones sociales } \\
\text { en riesgo }\end{array}$ & $\begin{array}{l}\text { Riesgo agregado } \\
\text { bajo }\end{array}$ & $\begin{array}{l}\text { Riesgo } \\
\text { incrementado }\end{array}$ & $\begin{array}{l}\text { Riesgo } \\
\text { incrementado }\end{array}$ & $\begin{array}{l}\text { Riesgo agregado } \\
\text { moderado }\end{array}$ & $\begin{array}{l}\text { Riesgo agregado } \\
\text { moderado }\end{array}$ & $\begin{array}{l}\text { Riesgo agregado } \\
\text { muy alto }\end{array}$ \\
\hline $\begin{array}{l}\geq 3 \mathrm{FR} \text {, Condiciones } \\
\text { sociales en riesgo o } \\
\text { sínd. metab o DBT }\end{array}$ & $\begin{array}{l}\text { Riesgo agregado } \\
\text { moderado }\end{array}$ & $\begin{array}{l}\text { Riesgo agregado } \\
\text { moderado }\end{array}$ & $\begin{array}{l}\text { Riesgo agregado } \\
\text { alto }\end{array}$ & $\begin{array}{l}\text { Riesgo agregado } \\
\text { alto }\end{array}$ & $\begin{array}{l}\text { Riesgo agregado } \\
\text { alto }\end{array}$ & $\begin{array}{l}\text { Riesgo agregado } \\
\text { muy alto }\end{array}$ \\
\hline $\begin{array}{l}\text { Condición clínica } \\
\text { asociada }\end{array}$ & $\begin{array}{l}\text { Riesgo agregado } \\
\text { alto }\end{array}$ & $\begin{array}{l}\text { Riesgo agregado } \\
\text { alto }\end{array}$ & $\begin{array}{l}\text { Riesgo agregado } \\
\text { muy alto }\end{array}$ & $\begin{array}{l}\text { Riesgo agregado } \\
\text { muy alto }\end{array}$ & $\begin{array}{l}\text { Riesgo agregado } \\
\text { muy alto }\end{array}$ & $\begin{array}{l}\text { Riesgo agregado } \\
\text { muy alto }\end{array}$ \\
\hline
\end{tabular}

Riesgo de enfermedad coronaria a 10 años: bajo $<10 \%$, moderado $10-19 \%$, alto $20-29 \%$, muy alto $>30 \%$. 
Tabla 2. Criterios de selección de farmacos.

\begin{tabular}{l}
\hline Propósito y tipo de evidencia: información versus prescripción \\
- Clasificación: ¿pre-hipertensión? \\
- Riesgo cardiovascular total: ¿riesgo absoluto versus relativo? \\
- ¿Cuandigación para órgano blanco: ¿qué investigar? \\
- Metas del tratamiento: ¿realmente debajo de 140/90 $\mathrm{mmHg}$ ? \\
\hline
\end{tabular}

Tabla 3. Fármacos preferidos para el tratamiento antihipertensivo.

\begin{tabular}{ll}
\hline $\begin{array}{c}\text { Daño subclínico a OB } \\
\text { HVI }\end{array}$ & BRAT, IECA, CA \\
Ateroesclerosis asintomática & CA, IECA, BRAT \\
Microalbuminuria & BRAT, IECA \\
Disfunción renal & BRAT, IECA \\
Evento clínico & \\
Previo ACV & Cualquier agente que disminuya PA (BRAT?,CA?) \\
Previo IMA & BB, IECA, BRAT \\
Angina pectoris & BB, CA \\
Falla cardiaca & Diuréticos, BB, IECA, BRAT, agentes antialdosterona \\
Fibilación auricular & \\
\multicolumn{1}{c}{ Recurrente } & BRAT, IECA \\
Permanente & BB, CA no-dihidropiridínico \\
IRCT/proteinuria & BRAT, IECA, diuréticos de asa \\
EVP & CA, BRAT/IECA \\
Condición & \\
HSA (adulto mayor) & Diuréticos, CA, BRAT \\
Sindrome metabólico & BRAT, IECA, CA(2) \\
Diabetes mellitus & BRAT, IECA \\
Embarazo & CA, metilldopa, BB \\
Negros & Diuréticos, CA \\
HVI: hipertrofia ventricular izquierda & ACV: accidente cerebrovascular IMA: infarto de miocardio agudo \\
HSA: hipertensión sistólica aislada & IRCT: insuficiencia renal \\
& \\
\hline
\end{tabular}

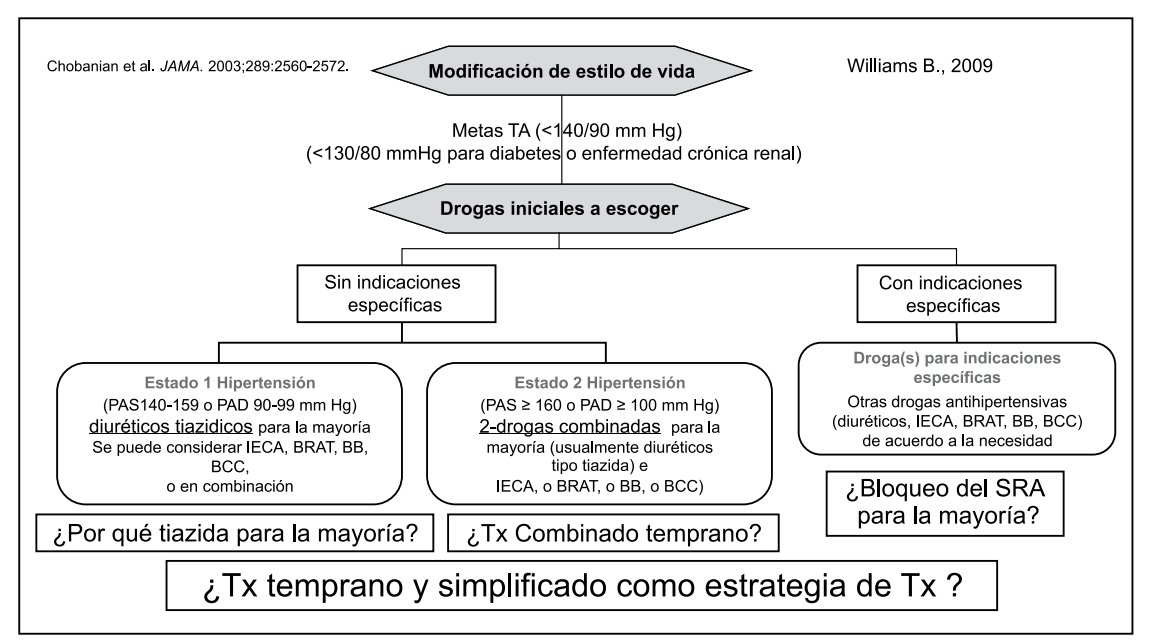

Figura 4. JNC 8. Algoritmo para el tratamiento de la hipertensión.
En pacientes con riesgo alto, principalmente diabéticos con enfermedad coronaria, se debe tener cuidado de no disminuir la presión arterial por debajo de 130-120/70-60 $\mathrm{mmHg}$, porque podría aumentar el riesgo de mortalidad cardiovascular, según recientes estudios ${ }^{(27)}$. En las guías para riesgo alto, se recomienda reducir la enfermedad cardiovascular y no solo la presión arterial. La presión arterial óptima sería 115/75 mmHg, y hay que tipificar al ateroesclerótico. Sin embargo, una PA menor de 120/70 mmHg puede exacerbar la curva J ${ }^{(27-29)}$. En este tipo de pacientes, considerar el tratamiento asociado con otros fármacos que controlen otros factores de riesgo, como la dislipidemia. De esta manera, el tratamiento combinado ayudaría a controlar mejor el riesgo global del paciente: presión arterial, colesterol LDL y proteína $\mathrm{C}$ reactiva ultrasensible ${ }^{(30)}$.

En conclusión, debemos considerar que ${ }^{(25,31)}$ :

- La enfermedad hipertensiva evoluciona a través del tiempo.

- El tratamiento debe estar direccionado a obtener un eficiente control de la presión arterial y regresión del daño a los órganos blanco.

- La terapia combinada es requerida para la mayoría de los pacientes, de manera de lograr el óptimo control de la presión arterial.

- El bloqueo del sistema renina angiotensina tiene fuerte evidencia sobre la base de reducir la presión arterial y protección de órganos blanco: debe ser el fundamento de la terapia antihipertensiva.

- La reducción total de la enfermedad cardiovascular incluye la modificación del estilo de vida, óptimo control de la presión arterial; terapia con estatinas y antiplaquetarios para los de riesgo alto, cuando sea apropiado.

- Las Guías de tratamiento antihipertensivo deben ser simples.

Consideramos que, en América Latina, la prevención cardiovascular no es una opción. Es una obligación. 
Tabla 4. Asociación de drogas antihipertensivas. II Consenso Latinoamericano de Hipertensión Arterial. 15-17 de mayo de 2008 - Asunción - Paraguay.

- La combinación de betabloqueadores con diuréticos predispone a la aparición de nuevos casos de diabetes y/o dislipidemia, por lo que no debería utilizarse en pacientes con síndrome metabólico o predisposición para diabetes.

- Las combinaciones de tiazidas con ahorradores de potasio previene la hipokalemia.

- El reciente estudio ACCOMPLISH informó mejores efectos en prevención de eventos con la asociación IECA-AC vs IECA-tiazidas.

- La combinación IECA-ARA II en algunos trabajos demostró disminución de la proteinuria. El estudio ON-TARGET no mostró mayores beneficios en prevención cardiovascular.

- Antes de asociar una tercera droga, esperar en lo posible 4 semanas hasta lograr efecto pleno de la asociación anterior, salvo en pacientes de riesgo alto.

Tabla 5. Previniendo la evolución de la enfermedad hipertensiva y riesgo cardiovascular.

- Disminuyendo las consecuencias metabólicas:

- Intervención en el estilo de vida

- Medicinas con 'amistad metabólica' (Bloqueo SRA)

- Estatinas

- Prevención del daño vascular estructural:

- Pequeñas arterias (resistencia vascular) (Bloqueo SRA)

- Grandes arterias (endurecimiento vascular) (Bloqueo SRA)

- Prevención del daño estructural cardio-renal:

- Hipertrofia ventricular izquierda (Bloqueo SRA)

- Disfunción sistólica y diastólica (Bloqueo SRA)

- Fibrilación auricular (Bloqueo SRA)

- Daño renal (Bloqueo SRA)

Es el bloqueo fundamental en el tratamiento moderno, de la hipertensión arterial.

Adap. Williams B. '09

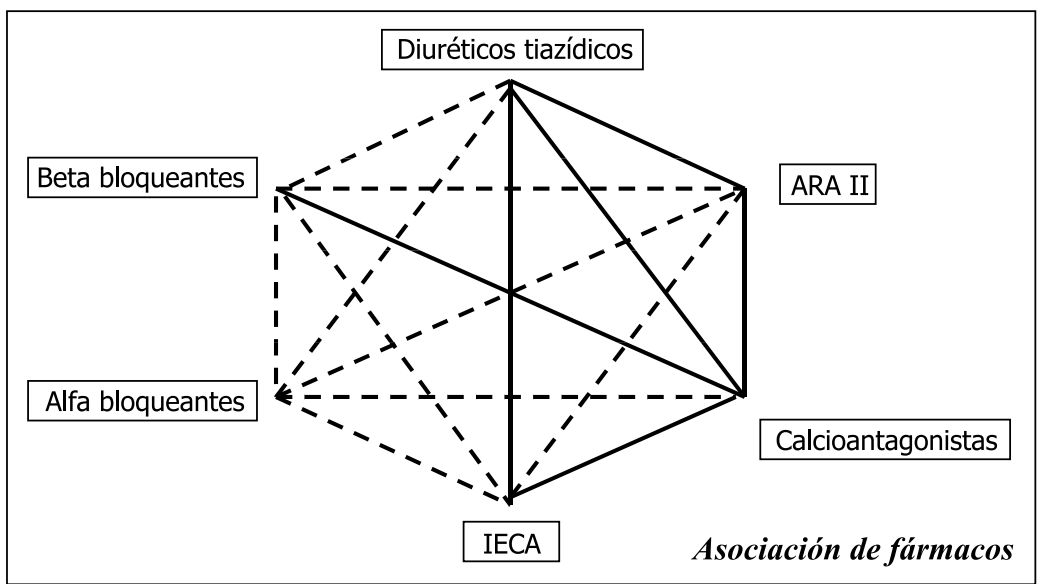

Figura 5. Combinaciones terapéuticas eficaces. Guías de Hipertensión Arterial ESC-ESH 2007.

\section{REFERENCIAS BIBLIOGRÁFICAS}

1. Jackson JH, Sobolski J, Krienke R, Wong KS, Frech-Tamas F, Nightengale B. Blood pressure control and pharmacotherapy patterns in the United States before and after the release of the Joint National Committee on the Prevention, Detection, Evaluation, and Treatment of High Blood Pressure (JNC 7) guidelines. J Am Board Fam Med. 2008;21(6):512-21.

2. MaJ, Lee KV, Stafford RS. Changes in antihypertensive prescribing during US outpatient visits for uncomplicated hypertension between 1993 and 2004. Hypertension. 2006;48(5):846-52.

3. Kaplan NM. The place of diuretics in preventing cardiovascular events. J Hum Hypertens. 2004;18 Suppl 2:S29-32.

4. Report of the Joint National Committee on Detection, Evaluation, and Treatment of High Blood Pressure. A cooperative study. JAMA. 1977;237(3):255-61.

5. Weir MR. Risk-based classification of hypertension and the role of combination therapy. I Clin Hypertens (Greenwich). 2008;10(1 Suppl 1):4-12.

6. Alderman MH. JNC 7: brief summary and critique. Clin Exp Hypertens. 2004;26(7-8):753-61.

7. Weber M, Basile J, Izzo J Jr, Oparil S. Roundtable discussion: blood pressure goal attainment: meeting the challenge of the JNC 7's blood pressure goals and the role of renin-angiotensinaldosterone system blockade. I Clin Hypertens (Greenwich). 2004;6(12):699-705.

8. Stergiou GS, Salgami EV; World Health OrganizationInternational Society of Hypertension (WHO-ISH); USA Joint National Committee on Prevention, Detection, Evaluation, and Treatment of High Blood Pressure (JNC-7); European Society of Hypertension-European Society of Cardiology (ESH-ESC). New European, American and International guidelines for hypertension management: agreement and disagreement. Expert Rev Cardiovasc Ther. 2004;2(3):359-68.

9. Rahn KH. [Recent intervention studies with antihypertensive drugs and their influence on guidelines]. Med Klin (Munich). 2003;98(12):771-5.

10. Chobanian AV, Bakris GL, Black HR, Cushman WC, Green LA, Izzo JL Jr, Jones DW, Materson BJ, Oparil S, Wright JT Jr, Roccella EJ; Joint National Committee on Prevention, Detection, Evaluation, and Treatment of High Blood Pressure. National Heart, Lung, and Blood Institute; National High Blood Pressure Education Program Coordinating Committee. Seventh report of the Joint National Committee on Prevention, Detection, Evaluation, and Treatment of High Blood Pressure. Hypertension. 2003;42(6):1206-52.

11. National High Blood Pressure Education Program. The Seventh Report of the Joint National Committee on Prevention, Detection, Evaluation, and Treatment of High Blood Pressure. Bethesda (MD): National Heart, Lung, and Blood Institute (US); Agosto 2004. 
Tabla 6. Terapia combinada en HTA. Fármacos.

- Preferidos: IECA/D; BRAT/D;IECA/BCC; BRAT/BCC

- Aceptables: BB/D; BB/BCC(DHP); BCC/D; IR/D; IR/BRAT; D/Ahorrador K

- Menos efectivos: IECA/BRAT; IECA/BB; BRAT/BB; BB/BCC(NDHP); BB/ Agentes centrales

Tabla 7. Terapia combinada en HTA. Sumario.

- Uso rutinario de terapia combinada para lograr las metas

- Utilizar combinaciones preferidas

- Utilizar rutinariamente en cifras mayores de 20/10 mmHg, para lograr metas

- Iniciar en el estadio I (a discreción del médico), especialmente cuando el segundo componente mejoraría el perfil de EA

- Mejor en una sola píldora (single pill combinations)

12. CushmanWC. JNC-7 guidelines: are they still relevant? Curr Hypertens Rep. 2007;9(5):380-6.

13. Inaba S, Iwai M, Tomono Y, Senba I, Furuno M, Kanno H, Okayama H, Mogi M, Higaki J, Horiuchi. M. Prevention of vascular injury by combination of an AT1 receptor blocker, olmesartan, with various calcium antagonists. Am J Hypertens. 2009;22:145-50.

14. Albert K. Hypertension in the oncology setting. Clin J Oncol Nurs. 2005;9(6):677-80. Erratum en: Clin J Oncol Nurs. 2006;10(1):16.

15. Devlin JW, Dasta JF, Kleinschmidt K, Roberts RJ, Lapointe M, Varon J, Anderson FA, Wyman A, Granger CB. Patterns of Antihypertensive Treatment in Patients with Acute Severe Hypertension from a Nonneurologic Cause: Studying the Treatment of Acute Hypertension (STAT) Registry. Pharmacotherapy. 2010;30(11):1087-96.

16. Andros V, Egger A, Dua U. Blood pressure goal attainment according to JNC 7 guidelines and utilization of antihypertensive drug therapy in MCO patients with type 1 or type 2 diabetes. J Manag Care Pharm. 2006;12(4):303-9.

17. Baruch L. Hypertension and the elderly: more than just blood pressure control. I Clin Hypertens (Greenwich). 2004;6(5):249-55.

18. Bezante GP, Viazzi F, Leoncini G, Ratto E, Conti N, Balbi M, Agosti S, Deferrari L, Deferrari G, Pontremoli R. Coronary flow reserve is impaired in hypertensive patients with subclinical renal damage. Am J Hypertens 2009;22(2):191-6.
19. Sanchez RA, Ayala M, Baglivo H, Velazquez C, Burlando G, Kohlmann 0, Jimenez J, López Jaramillo P, Brandao"A, Valdes G, Alcocer L, Bendersky M, Ramirez AJ, Zanchetti A, on behalf of the Latin America Expert Group. Latin American guidelines on hypertension. J Hypertens.2009;27(5):90522.

20. Milic M, Ziegler MG. Discrepant antihypertensive dose recommendations. Clin Exp Hypertens. 2006;28(2):171-80.

21. Moser M. Rationale for combination therapy in the management of hypertension. I Clin Hypertens (Greenwich). 2003;5(6 Suppl 4):17-25.

22. ESH/ESC Hypertension Practice Guidelines Committee. Practice guidelines for primary care physicians: 2007 ESH/ESC hypertension guidelines. J Hypertens. 2007;25:1105-87.

23. Welch V, Tang SS. Treatment and control of BP and lipids in patients with hypertension and additional risk factors. Am J Cardiovasc Drugs. 2007;7(5):381-9.

24. Di Martino M, Esposti LD, Filigheddu F, Veronesi C, Salerno G, Saragoni S, Glorioso N, Didoni G, Esposti ED. Use of antihypertensive and lipidlowering drugs: the management of cardiovascular risk in clinical practice. J Hum Hypertens. 2007;21(1):53-9.

25. Williams $B$. The changing face of hypertension treatment: treatment strategies from the 2007 ESH/ESC hypertension Guidelines. J Hypertens Suppl. 2009;27(3):S19-26.
26. Gradman AH, Basile JN, Carter BL, Bakris GL; American Society of Hypertension Writing Group. Combination therapy in hypertension. J Am Soc Hypertens. 2010;4(1):42-50.

27. Bangalore S, Messerli FH, Wun CC, Zuckerman AL, Demicco D, Kostis JB, Larosa JC; Treating to New Targets Steering Committee and Investigators. J-curve revisited: an analysis of blood pressure and cardiovascular events in the Treating to New Targets (TNT) trial. Eur Heart J. 2010 Sep 16. [Publicación electrónica antes de impresión].

28. Nilsson P. The highlights from the Oslo Hypertension Meeting now available! Editor's page. Disponible en: http://www.eshonline.org/Publications/ editorspick.aspx. Obtenido el 29 se setiembre de 2010.

29. Brookes L. Hipertension highlights: update for European Guidelines, plus new evidence for blood pressure targets and strategies. Medscape Cardiology. Disponible en: http://www.medscape. com/viewarticle/710127. Obtenido el 29 de setiembre de 2010.

30. Ismail-Beigi F, Craven T, Banerji MA, Basile J, Calles J, Cohen RM, Cuddihy R, Cushman WC, Genuth S, Grimm RH Jr, Hamilton BP, Hoogwerf B, Karl D, Katz L, Krikorian A, O'Connor P, Pop-Busui R, Schubart U, Simmons D, Taylor H, Thomas A, Weiss D, Hramiak I; ACCORD trial group. Effect of intensive treatment of hyperglycaemia on microvascular outcomes in type 2 diabetes: an analysis of the ACCORD randomised trial. Lancet. 2010;376(9739):419-30.

31. Muntner P, Krousel-Wood M, Hyre AD, Stanley E, Cushman WC, Cutler JA, Piller LB, Goforth GA, Whelton PK. Antihypertensive prescriptions for newly treated patients before and after the main antihypertensive and lipid-lowering treatment to prevent heart attack trial results and seventh report of the joint national committee on prevention, detection, evaluation, and treatment of high blood pressure guidelines. Hypertension. 2009;53(4):617-23.

\section{Correspondencia:}

Dr. Alfonso Bryce Moncloa

Clínica El Golf

Av. Aurelio Miró Quesada $10505^{\circ}$ piso San Isidro Correo electrónico: cardiogolf1@yahoo.es 